\title{
LA ENSEÑANZA DE LA FÍSICA PARA LA FORMACIÓN DE PROFESORES DE FÍSICA ${ }^{1}$
}

\author{
María Mercedes Ayala M.
}

\begin{abstract}
That one of the main task for the science teacher is the recontextualization of the different theories and that the character of teachers activity is cultural, is put fotward in this paper.
\end{abstract}

\section{RESUMEN}

Basados en las reflexiones y desarrollos del Programa de Maestría en Docencia de la Física y en algunas experiencias del Programa de Licenciatura de Física de la Universidad Pedagógica Nacional de Colombia, en el presente escrito se plantea como campo de trabajo del docente de ciencias la recontextualización de los saberes científicos, se pone de manifiesto el carácter eminentemente cultural de dicha actividad y la complejidad de la misma. Tal enfoque implica superar las concepciones sobre la ciencia y sobre la enseñanza que hoy animan gran parte de los programas de formación de los maestros de ciencias. Por último, se plantea la pregunta por el tipo de formación en física que debe tener un profesor de física para estar en capacidad de orientar los procesos de recontextualización de los sabores científicos, y se dan algunos elementos en este sentido.

\section{ALGUNAS CONCEPCIONES VIGENTES ACERCA DE LA FORMACIÓN DE MAESTROS}

Al ser la relación con los productos de la ciencia la forma más generalizada e inmediata de ponerse en contacto con la empresa científica -yen mayor grado en países como el nuestro, que, reducidos a la calidad de consumidores de los mismos, permanecen ajenos a la actividad de producción científica-, no es de extrañar que se tienda a considerar la enseñanza de las ciencias como una actividad de transmisión de saberes, técnicas y procedimientos científicos. Tampoco es de extrañar que las deficiencias en el manejo por parte del maestro de la disciplina que enseña sea el problema que con más frecuencia y énfasis se señala con relación a la formación de los docentes; así el término "un manejo adecuado de la disciplina" no tenga un significado suficientemente preciso; con éste se puede referir a la cantidad de información, o a la actualización de la misma, o a las

\footnotetext{
1 Trabajo presentado en la $\mathrm{V}$ Reunión Latinoamericana sobre Enseñanza de la Física, Porto Alegre (Gramado). Agosto 24 a 28 de 1992.

Profesora Asociada Universidad Pedagógica Nacional. Departamento de Física. e-mail: ayalam@uni.pedagogica.edu.co 
habilidades en la resolución de problemas o en la realización de experimentos, o a otros aspectos menos comunes.

Tradicionalmente y desde esta forma de asumir las ciencias dos ideas con diversos matices se han venido manejando y desarrollando en torno a la formación de maestros de ciencias. $^{2}$

Para unos, un conocimiento adecuado de lo que ha de ser enseñado es la garantía de la buena enseñanza: quien sabe física sabe enseñar física; más aún quien se forma como investigador en física asegura una mejor formación como maestro de física.

Para otros, reconociendo que la práctica pedagógica supone un campo de acción que trasciende el saber de la disciplina o disciplinas que han de ser enseñadas, ven que se requiere además un saber propio de la profesión de maestro. Saber práctico y teórico alimentado por diferentes disciplinas científicas: lo pedagógico; que se refiere usualmente al acerbo de métodos y modelos que pueden usarse en los procesos de transmisión de saberes, a la fundamentación de los mismos -casi siempre en teorías de corte sicológico-, y, también en algunos casos, a un cierto conocimiento de la problemática educativa en general y de las políticas educativas del país.

La enseñanza de las ciencias se plantea, entonces, como un campo de trabajo que le exige al maestro dos competencias: una en el saber pedagógico y la otra en la disciplina que ha de ser enseñada. Pero como es de esperar, difícilmente se logra la concreción de esta perspectiva: se termina formando al maestro con algún conocimiento de ciencias y alguno de pedagogía, y con grandes problemas de identidad profesional. Por un lado, asumir la ciencia como la colección de resultados de la actividad científica, convierte a los contenidos en el eje central de la enseñanza e introduce una problemática de muy difícil solución cuando se trata de garantizar que el maestro tenga un conocimiento adecuado de la disciplina que debe enseñar; puesto que la selección y tratamiento de los mismos están sometidos a tres criterios mutuamente contradictorios: extensión, profundidad y actualización. Por otro lado, la pedagogía general es aún un campo intelectual amorfo con un desarrollo muy incipiente en el que no se han dado todavía procesos de acumulación de conocimientos y constitución de tradiciones ${ }^{3}$ en lo que se refiere a los resultados de la investigación en enseñanza de las ciencias, que se ha venido desarrollando con especial énfasis en la última década, su introducción en los cursos de pedagogía y didáctica se ha venido dando de manera fragmentaria y lenta.

No obstante las dificultades que la yuxtaposición del saber disciplinar y el saber pedagógico plantea para los programas de formación de docentes en ciencias, esta concepción ha inspirado la mayoría de los diseños y desarrollos curriculares de los programas de formación de docentes de ciencias en el país; más aún, inspira al proyecto de ley sobre las instituciones formadoras de docentes que actualmente cursa en el Congreso. Buscando generar las condiciones para que el manejo de estas dos competencias por los maestros de ciencias del nivel medio sea posible, se propone que el maestro obtenga primero su título universitario en la disciplina que ha de enseñar (5 años) y posteriormente se especialice en pedagogía (1 año), opción que a pesar de haberse seguido en muchos países no ha permitido superar los múltiples problemas involucrados en la enseñanza de la física o de las ciencias en general.

\footnotetext{
2 A este respecto, resulta ilustrativo el capítulo 4E del informe intitulado "L'enseignement aujourd'hui fonctions, status, politiques", publicado por la OCDE, (Paris, 1990).

3 MOCKUS, A. Lugar de las Pedagogías en las Universidades Interaulas, serie pedagógica № 3, Universidad Nacional de Colombia.
} 
Pero el problema no radica en las dificultades de organizar y poner en práctica un programa de formación que garantice por parte del maestro un manejo de las dos competencias anteriormente mencionadas; el problema radica en los supuestos que con relación a la ciencia, al papel que juega su enseñanza en la educación básica y media y al carácter de dicha actividad, animan esta concepción.

\section{DE LA TRANSMISIÓN DE CONTENIDOS DE FÍSICA A LA RECONTEXTUALIZACIÓN DE LOS SABERES DE LA FÍSICA: REDEFINICIÓN DE LA ACTIVIDAD DEL MAESTRO}

La disociación entre la actividad científica y sus productos ha llevado a asumir la ciencia como un cuerpo de hechos, conceptos, leyes, teorías y técnicas, etc; convirtiendo a "los contenidos" - como ya hemos dicho- en el eje de la enseñanza. Dado el acelerado desarrollo de las ciencias en el presente siglo, tal enfoque ha introducido una problemática de muy difícil solución, que reviste características especiales en el caso de la enseñanza de la física.

Es así, como en ja década de los 60 ante las dificultades planteadas por ja gran cantidad y rápido crecimiento de los contenidos se optó, en un caso, por desplazar el énfasis en la enseñanza de la física de los contenidos hacia "el método", centrándola en el trabajo de laboratorio - perspectiva asumida por el PSSC, por ejemplo- y, en otros, por hacer presentaciones más estructuradas de los contenidos -como fue el caso del proyecto BERKELEY.

En la actualidad el problema sigue vigente. John S. Ridgen, por ejemplo, en el editorial del American Journal of Physics de octubre de 1985, reta a la comunidad de los físicos, y en especial a los profesores universitarios, a reestructurar de manera radical los cursos introductorios de física, de modo que los estudiantes logren a través de éstos tener un panorama de la actividad contemporánea de la física. A pesar de la gran dinámica de la física — dice Ridgen- ésta no es reflejada en sus cursos introductorios; su contenido ha permanecido estático durante un siglo.

A modo de respuesta al reto planteado por Ridgen, R. Hilbron señala, en su artículo "Redesigning college and university level introductory physics" ${ }^{4}$, que la fuente del problema radica en las ideas, ampliamente compartidas por los profesores de física, de "cubrir contenidos" y de que es necesario seguir la cronología histórica del desarrollo de la disciplina: se considera que "no es posible entender la mecánica cuántica o la relatividad sin un completo entendimiento de la mecánica newtoniana, las ondas, la teoría electromagnética, la termodinámica, etc.". La superación de tal concepción es requerida, entonces, para hacer posible la elaboración de nuevas selecciones y estructuraciones de los contenidos de física a enseñar.

En este sentido, el Instituto de Didáctica de la Física de la Universidad de Karlsruhe ha venido elaborando un proyecto desde comienzos de la década de los 80, en el que, buscando hacer la enseñanza de la física más eficiente desde el punto de vista disciplinar -es decir, buscando generar condiciones para que los estudiantes accedan rápidamente a las formas actuales de ver los fenómenos físicos-se hace una profunda transformación de la física que se enseña.

\footnotetext{
${ }^{4}$ HILBORN, R. "Redesigning college and university level introductory physics". Am. J. Phys., Vol. 56, No. 1, 1988.
} 
Se parte de una crítica a la tradición que toma a la mecánica newtoniana como la temática con la que se inicia el estudio de la física. Tradicionalmente - dice G. B. Schmid en un artículo donde hace una presentación del proyecto ${ }^{5}$ - la mecánica está en el corazón de la enseñanza de la física: un problema físico se considera que está entendido, en principio al menos, si puede ser explicado en términos mecánicos, es decir, en términos de los movimientos de masas puntuales y de sus interacciones. A pesar de que la relatividad y la cuántica suponen nuevas formas de abordar los fenómenos físicos, $y$, más aún, de que la cuántica excluye el concepto clásico primario de partícula como masa puntal que puede seguirse a través del espacio y del tiempo, esta imagen newtoniana del mundo se sigue enseñando. Las cantidades predominantes en mecánica cuántica tales como energía, momentum, momento angular, carga eléctrica y número de partículas prosigue Schmidson más similares a las cantidades extensivas de la termodinámica que a los conceptos básicos newtonianos: trayectoria, velocidad, masa, y fuerza. Si bien cantidades como energía, momentum y momento angular aparecen en la mecánica de Newton, éstas se presentan como instrumentos útiles para el cálculo y no como conceptos primarios. El énfasis en esta visión - dice Schmid- resulta contraproducente para una comprensión adecuada de la física actual. Por estas razones, el proyecto de física de Karlsruhe contra la enseñanza de la física en cantidades del mismo tipo de las cantidades básicas de la mecánica cuántica así como de las cantidades extensivas de la termodinámica.

La realización de un proyecto de este corte involucra no solo la superación de las dos ideas que Hilbron menciona como los obstáculos que impiden hacer nuevas selecciones y estructuraciones de los contenidos objeto de enseñanza, sino también la superación de otra, muy arraigada en la comunidad de maestros de física y de ciencias en general: no afectar ni permitir que los estudiantes afecten el contenido científico que se enseña. La separación del producto de la actividad científica lleva necesariamente a la "cosificación" de los contenidos científicos. El proyecto de Karlsruhe es buen ejemplo de la acción consciente y fundamentada de transformación de los contenidos científicos que se requiere para hacer propuestas verdaderamente innovadoras en el campo de la enseñanza de la física.

Pero el problema exige la consideración de otro aspecto que es fundamental, anotado también por Hilbron en el artículo arriba mencionado y por investigadores en la enseñanza de la física como Paolo Guidoni de la Universidad de Nápoles, y que es claramente manifiesto en los diversos trabajos que se han venido realizando en el presente siglo sobre la historia de las ciencias: la física más que un cuerpo de hechos, conceptos y teorías, es un proceso y una actividad. Se requiere - dice Hilbron- destacar en la enseñanza las preguntas que los físicos hacen a la naturaleza y los métodos usados para contestar esas preguntas; enfrentar el aspecto de cómo esas preguntas y respuestas cambian con el desarrollo de nuevas teorías y con los nuevos descubrimientos experimentales, en fin, mostrar el carácter dinámico de la física.

Sin duda alguna destacar el planteamiento y reformulación de los problemas que han posibilitado la construcción de fenómenos, la formación y desarrollo de los conceptos y su sistematización en teorías, permite visualizar la ciencia como un proceso y más aún como una actividad humana. Pero asumir la ciencia como actividad significa algo más; remite entre otras cosas a preguntarse por la pertinencia de los problemas planteados y por los intereses a los que responde, por las condiciones en las cuales una actividad de conocimiento en una época concreta es considerada científica, por la legitimidad que ésta

${ }^{5}$ SCHMID, G. B. "An up-to-date approach physics". Am. J. Phys. 52(9), 1984. 
tiene para la sociedad, por los procesos de difusión de los nuevos sabores en la comunidad de especialistas así como en sectores más amplios de la población.

El gran auge que han tomado en la última década las teorías constructivistas del conocimiento individual, ha destacado un aspecto adicional, que hace que la problemática de la enseñanza de la física se torne aún más compleja: el conocimiento individual es un proceso. Y ello significa - dice Guidoni- que el conocimiento es diferente silos procesos lo son, así los resultados o productos sean análogos; por eso considera que no se debe confundir una buena formación en ciencias con el aprendizaje y manejo de ciertos argumentos, tan correctos técnicamente como un profesor sea capaz de transmitir.

Desde este punto de vista, al proyecto de Karlsruhe se le puede hacer una importante objeción: se trata de que el estudiante se apropie y se adiestre en una sola manera de abordar el mundo físico — forma que, como ya lo dijimos_, permite un acceso más eficiente a la problemática de la física contemporánea. Precisamente, esa actividad de elaboración constante de una mejor comprensión del mundo, esa experiencia de conocimiento, parece ser omitida en este proyecto.

Por otro lado, si se analizan las tendencias constructivistas en la enseñanza de las ciencias, es importante notar que una buena parte de las propuestas de innovación y trabajos de investigación de este corte están limitados por la concepción de ciencia que manejan; mantienen la misma relación de exterioridad con los contenidos a enseñar que las formas más tradicionales de enseñanza; sin embargo, el hecho de asumir el conocimiento individual como un proceso de construcción de esquemas conceptuales plantea nuevas formas de abordar la enseñanza de las ciencias desde la perspectiva metodológica.

Dado que todas las actividades de los individuos y colectividades está signada por los valores culturales que han sido interiorizados por éstos y que tanto la ciencia como el conocimiento individual son actividades, la enseñanza de las ciencias tiene un carácter eminentemente cultural.

Los aspectos hasta aquí contemplados delimitan en buena parte la problemática de la recontextualización de los sabores científicos, que es precisamente el campo de trabajo de los profesores de ciencias.

Para tener una mejor comprensión de la complejidad del trabajo involucrado en la recontextualización de los sabores científicos, es necesario hacer una consideración adicional. La intencionalidad de la acción pedagógica o, más precisamente, el sentido que se le dé a la enseñanza de las ciencias en contextos específicos establece una directriz y se constituye en el motor de dicha actividad. Así, preguntarse por el sentido de la enseñanza de las ciencias en los niveles básicos remite a preguntarse por la formación en ciencias que debe tener un ciudadano y por su importancia. En países como el nuestro -ajenos a la actividad de producción científica, pero cada vez más impactada por ésta- la legitimación de dicha actividad ante la sociedad se constituye en un problema crucial para la enseñanza de las ciencias.

En las condiciones actuales de internacionalización de la economía y de la cultura y de competencia en el campo del conocimiento, se impone como indispensable el desarrollo científico y tecnológico del país. Pero ello requiere -como ha sido reconocido en los diversos estudios que están inspirando en la actualidad las políticas nacionales de ciencia 
y tecnología- lograr que la ciencia se arraigue en nuestra cultura ${ }^{6}$. Una cultura científica asumida por amplios sectores de la población es, pues, condición de posibilidad de la actividad científica en el país. Y esto implica cambiar la relación que los individuos y colectividades mantienen con el conocimiento científico.

Precisamente, la posibilidad de legitimación o no de la actividad científica por la sociedad reside en la forma como sea apropiada la ciencia por la cultura de base: la mitificación de la ciencia - debida en gran medida a la relación de exterioridad que se mantiene con el conocimiento científico y a la inevitable perplejidad que causan los productos tecnológicos cuando no se comprenden los principios que fundamentan su producción y funcionamiento- es quizás el mayor obstáculo para el desarrollo de la ciencia y de la tecnología en el país.

Es importante tener en cuenta - como dice Paolo Guidoni- que nada se puede transferir a una cultura sin que sea transformado por ella; para que algo adquiera significado y pueda ser valorado requiere ser puesto en términos de los conocimientos existentes y su organización en el contexto cultural en cuestión. Son las estructuras internas de la cultura común y sus articulaciones las que conforman una base fundamental para el desarrollo, significado y coordinación de todas las subculturas particulares, entre ellas las científicas. Así, un proceso de asimilación cultural significa una transformación tanto de aquello que es asimilado como de aquello que lo asimila.

En este orden de ideas, la enseñanza de las ciencias en los niveles básico y medio debe:

- Favorecer una mejor comprensión del mundo en el cual la ciencia y la tecnología constituyen elementos esenciales del desarrollo y en el cual los productos de la actividad científica están permanente presentes en los diversos ámbitos de la vida de los individuos.

- Posibilitar que el conocimiento científico sea valorado en su justa dimensión por la sociedad en general. Posibilitar la participación ciudadana en las decisiones que sobre los productos y la empresa científica se deban tomar en el país.

- Generar condiciones para que nuestra cultura participe en los procesos de producción científica.

El sentido de la enseñanza de las ciencias planteado en estos términos enfatiza aún más el carácter cultural de la actividad del profesor de ciencias.

\section{LA FORMACIÓN EN FÍSICA REQUERIDA PARA LA ORIENTACIÓN DE PROCESOS DE RECONTEXTUALIZACIÓN DE LOS SABERES DE LA FÍSICA}

Hasta el momento se ha dado una visión panorámica de la actividad del docente de ciencias en los niveles básico y medio. Surge, entonces, la pregunta sobre el tipo de formación que el profesor de física debe tener para orientar los procesos de recontextualización de este campo del saber. Es importante notar que tal problemática

\footnotetext{
6 Véase al respecto: "Ciencia y Tecnología para una Sociedad Abierta", COLCIENCIAS, Instituto Colombiano para el Desarrollo de la Ciencia y la Tecnología, Departamento de Planeación Nacional; y "Estructura Científica Desarrollo Tecnológico y Entorno Social", editado por el Departamento Nacional de Planeación: Misión de Ciencia y Tecnología, Santa Fe de Bogotá D.C., 1990.
} 
supone la consideración de muchos aspectos dada la complejidad de su quehacer. Nos limitaremos en este documento a examinar cuál debe ser el conocimiento de la física que debe tener un docente de física para orientar dichos procesos.

En primer lugar, podemos afirmar, a la luz de la concepción sobre el quehacer del docente de ciencias arriba planteada, que un profesor de física requiere una sólida formación en esta disciplina, pero decididamente una formación diferente a la que se le da a un físico; y no porque sea de menor calidad sino porque ha de formarse para desarrollar un trabajo de intervención cultural. Esta diferencia de intereses y de sentido que hay entre la formación de un físico y de un profesor de física, así como sus implicaciones han comenzado a ser reconocidas. J. S. Ridgen, por ejemplo, en el editorial del American Journal of Physics de agosto de 1986, comentando las recomendaciones del "Carnigie report: A Nation Prepared: Teachers for the 2lst Century", señala esta diferencia de intereses como una gran fuente de dificultades cuando las facultades de ciencias asumen, como lo propone el mencionado reporte, la formación de los maestros de ciencias. El problema de la educación —dice Ridgen - son los procesos cognitivos. Que no es añadimos - la preocupación central de los físicos.

Se requiere pues un enfoque diferente en la formación en física para los maestros de física. Tal formación debe, además de darle al maestro elementos válidos para la recontextualización de los sabores de la física, generar en él una actitud de continua indagación sobre los procesos del conocimiento en la física.

Conocer la física para un profesor de física es conocer, en primer lugar, los problemas que han posibilitado la construcción de fenómenos, la formación y el desarrollo de los conceptos de la física y su sistematización en teorías; las condiciones en que tales problemas fueron planteados, en especial, su pertinencia en el momento histórico en que fueron formulados.

En segundo lugar, conocer la física significa estar en capacidad de caracterizar los procesos de diferenciación conceptual que se desarrollan en la construcción de nuevos fenómenos, en la redefinición y reconceptualización de una problemática, yen la constitución de nuevos objetos disciplinares y métodos y técnicas para abordarlos. Significa también estar en capacidad de caracterizar las diversas explicaciones involucradas en estos procesos.

En tercer lugar, conocer la física significa, también, vivenciar procesos de construcción de fenómenos y de conceptos y de elaboración de explicaciones. Tal experiencia de conocimiento resulta vital para el maestro de física; es condición necesaria para que el maestro pueda encaminar los procesos que lleven al estudiante a darse explicaciones acordes con los desarrollos científicos.

En último lugar, conocer la física es conocer los procesos a través de los cuales se difunden y legitiman las ideas y acciones de la comunidad de los físicos en los contextos históricos particulares, condición necesaria para que la sociedad provea los recursos necesarios para el desarrollo de la disciplina. 